\title{
An experimental evaluation with Drosophila melanogaster of a novel dynamic system for the management of subdivided populations in conservation programs
}

\author{
V Ávila ${ }^{1}$, J Fernández ${ }^{2}$, H Quesada ${ }^{1}$ and A Caballero ${ }^{1}$

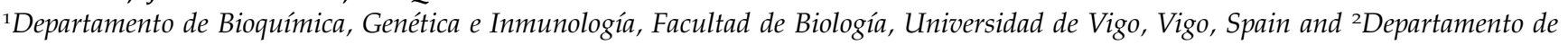 \\ Mejora Genética, Instituto Nacional de Investigación y Tecnología Agraria y Alimentaria, Madrid, Spain
}

\begin{abstract}
A dynamic method (DM) recently proposed for the management of captive subdivided populations was evaluated using the pilot species Drosophila melanogaster. By accounting for the particular genetic population structure, the DM determines the optimal mating pairs, their contributions to progeny and the migration pattern that minimize the overall coancestry in the population with a control of inbreeding levels. After a premanagement period such that one of the four subpopulations had higher inbreeding and differentiation than the others, three management methods were compared for 10 generations over three replicates: (1) isolated subpopulations (IS), (2) onemigrant-per-generation rule (OMPG), (3) DM aimed to produce
\end{abstract}

the same or lower inbreeding coefficient than OMPG. The DM produced the lowest coancestry and equal or lower inbreeding than the OMPG method throughout the experiment. The initially lower fitness and lower variation for nine microsatellite loci of the highly inbred subpopulation were restored more quickly with the DM than with the OMPG method. We provide, therefore, an empirical illustration of the usefulness of the DM as a conservation protocol for captive subdivided populations when pedigree information is available (or can be deduced) and manipulation of breeding pairs is possible.

Heredity (2011) 106, 765-774; doi:10.1038/hdy.2010.117; published online 8 September 2010

Keywords: genetic diversity; inbreeding; one-migrant-per-generation rule; population structure; microsatellite loci

\section{Introduction}

Most populations of domestic and wild animal and plant species at risk of extinction are spatially structured. This structuring must be explicitly considered in the conservation management of genetic resources and in the fields of animal breeding and evolutionary biology. For instance, most species undergoing conservation programs in captivity are generally maintained in independent nuclei (zoos, botanic gardens, germplasm centers and so on) (Valladares-Padua et al., 2002; Watanabe et al., 2009). In some situations, subdivision has a clear biological meaning, as different subpopulations are characterized by local adaptations (Storfer, 1999; Branch et al., 2003). This may be, for example, the case of domestic breeds or strains, in which separation is desirable in order to keep a required level of morphological differentiation. The structuring of populations in reduced and, sometimes, isolated groups, has an impact on the erosion of genetic variation and the increase in inbreeding depression, factors of importance in conservation programs (Frankham et al., 2002). It is, therefore,

Correspondence: Dr V Ávila, Departamento de Bioquímica, Genética e Inmunología, Facultad de Biología, Universidad de Vigo, Vigo, Pontevedra 36310, Spain.

E-mail:vavila@uvigo.es

Received 7 October 2009; revised 27 July 2010; accepted 30 July 2010; published online 8 September 2010 needed to establish methods for the management of genetic variation in subdivided populations and to look for objective criteria to quantify the importance of within- versus between-subpopulation variation.

The genetic diversity of a population is usually quantified by its expected heterozygosity or gene diversity (Nei, 1973), or through its complementary, the average coancestry (kinship). For a single population under random mating, the average coancestry equals the average inbreeding coefficient in the following generation. For a subdivided population, however, a distinction between coancestry and inbreeding is useful, as the rate of increase in inbreeding and coancestry, or equivalently the inbreeding and variance effective population size, can be different (Wang and Caballero, 1999). The global coancestry in a structured population provides a measure of the global gene diversity and, therefore, the evolutionary potential of the whole population. In contrast, the average inbreeding provides a measure of the local homozygosity in each subpopulation.

Controlling the rate of increase in inbreeding and coancestry provides a general framework for managing genetic resources in conservation programs. The control of inbreeding and coancestry would restrict inbreeding depression, the probability of losing rare alleles and the risk of extinction (Frankham et al., 2002). The realistic options for managing a captive breeding program are influenced by the understanding and development of selection and mating strategies for restricting the 
increase of inbreeding (for example, Ballou and Lacy, 1995; Meuwissen, 2007; Toro et al., 2009). In the development of captive breeding programs, two decisions need to be made: how widely and which individuals should contribute to the progeny and how these individuals should be mated. When the pedigree of the population is available, parent contributions (number of offspring breeding in the next generation) should be optimized by minimizing the global coancestry weighted by those contributions (Haig et al., 1990; Ballou and Lacy, 1995). Minimun coancestry (1) maximizes expected heterozygosity and effective population size (Caballero and Toro, 2000, 2002), (2) is flexible and robust against departures from the ideal conditions (Fernández et al., 2003), (3) minimizes the loss of alleles (Fernández et al., 2004) and (4) preserves the original distribution of allele frequencies (Saura et al., 2008). The minimum-coancestry mating system can be applied subsequently to selected individuals resulting from the optimization (Caballero et al., 1996; Sonesson and Meuwissen, 2000; Meuwissen, 2007). However, algorithms are also available to optimize simultaneously the mating scheme and parent contributions, the so-called mate selection (Toro and Pérez-Enciso, 1990; Fernández et al., 2001).

Maximum gene diversity (minimum global coancestry) of a population in the long term is attained by subdividing it in as many isolated groups as possible (Kimura and Crow, 1963; Robertson, 1964; Wang and Caballero, 1999), as different allelic variants will get fixed in each group, becoming a genetic reservoir of variation. However, complete isolation leads to increased rates of local inbreeding with the possible consequence of inbreeding depression. Thus, a certain degree of gene flow should be maintained through migration of individuals between subpopulations (Allendorf and Luikart, 2007). A commonly accepted rule of thumb, based on the island model derived by Wright (1931), establishes that one-migrant-per-generation (OMPG) per subpopulation is needed to maintain considerable differentiation between subpopulations, while avoiding an excessive increase in inbreeding (Mills and Allendorf, 1996; Wang, 2004). However, a critical characteristic of this simple management method is that the particular migration scheme used is independent of the genetic structure of the population, ignoring the pedigree relationships across subpopulations.

The management of captive subdivided populations has not been considered in the conservation of genetic resources until recently. Wang (2005) proposed a method to optimize the global genetic diversity controlling the rate of inbreeding from demographic information. Fernández et al. (2008) extended this idea for the situation in which genealogical information is available, by developing a dynamic methodology, which allows for an efficient use of pedigree records. The dynamic method (DM), which can be implemented by a free software (METAPOP; PérezFigueroa et al., 2009), allows for obtaining the lowest levels of average coancestry (maximal gene diversity) with specific restrictions in the level of subpopulation inbreeding by means of the establishment of optimal contributions from individuals and specific migrations between subpopulations. The methodology of Fernández et al. (2008) was evaluated with computer simulations and outperformed the OMPG rule by producing less inbreeding and more gene diversity than the OMPG method.
Here, we evaluate the performance of the DM in minimizing coancestry and inbreeding relative to the OMPG method in subdivided experimental populations of Drosophila melanogaster.

\section{Materials and methods}

\section{Base population and culture conditions}

More than 1000 individual D. melanogaster were collected in a wine cellar close to Vigo (northwest Spain) in November 2006. Thirty bottles with 30-60 individuals each (base population) were founded and maintained with circular mating until the start of the experiment in February 2008. Flies were reared in a culture medium composed of 11 water, $100 \mathrm{~g}$ brewer's yeast, $100 \mathrm{~g}$ sucrose, $12 \mathrm{~g}$ agar, $2.5 \mathrm{~g} \mathrm{NaCl}$ and $5 \mathrm{ml}$ propionic acid and were handled at room temperature under $\mathrm{CO}_{2}$ anesthesia. All cultures were incubated in a chamber at $25 \pm 1{ }^{\circ} \mathrm{C}, 65 \pm 5 \%$ relative humidity and maintained under continuous lighting. Virgin males and females were used for mating across the entire experiment. A $\operatorname{In}(2 \mathrm{LR}) \mathrm{CyO}, \mathrm{Cy} / \mathrm{L}^{2}$ commercial balancer stock was used to estimate competitive fitness $(c w)$. The stock flies carry the Cy (Curly wings) and $L^{2}$ (small Lobe eyes) genes. These mutations are both located on the second chromosome and have dominant and conspicuous phenotypic effects, which make easier to distinguish stock flies from wild-type flies. This stock was maintained in 10 bottles each with ca. 100 individuals throughout the experiment.

\section{Experimental design}

To evaluate the performance of the DM in comparison with other strategies, we generated a structured population composed of four subpopulations with different levels of inbreeding and differentiation among them. The experiment consisted of (1) a pre-management period to replicate what might be found at the beginning of a genetic-management program in captivity and (2) a management period in which monogamous pairings of individuals were manipulated at each generation according to different methodologies.

Pre-management period: Experimental populations were established seven generations (generation -7 to -1 ) before management in order to generate a population subdivided into four subpopulations of size $N=16$, where one of them shows a higher inbreeding level than the others and a substantial genetic differentiation with the rest. Ninety-six couples were randomly chosen from the base population to establish 3 replicates of 32 couples/vials each. The scheme followed for each of these replicates is shown in Figure 1. In order to generate a subpopulation more inbred and differentiated than the others, two randomly chosen groups, one formed by $N=16$ individuals (eight couples in eight vials) and another by $N=48$ individuals ( 24 couples in 24 vials) were maintained separately with discrete generations, random mating between pairs and Poisson's contributions from couples to the progeny (obtained from a Poisson's probability table). After this period, the first group became one of the subpopulations (eight couples), and the second group was randomly divided into the remaining three subpopulations of eight couples each. This protocol resulted in that the former 


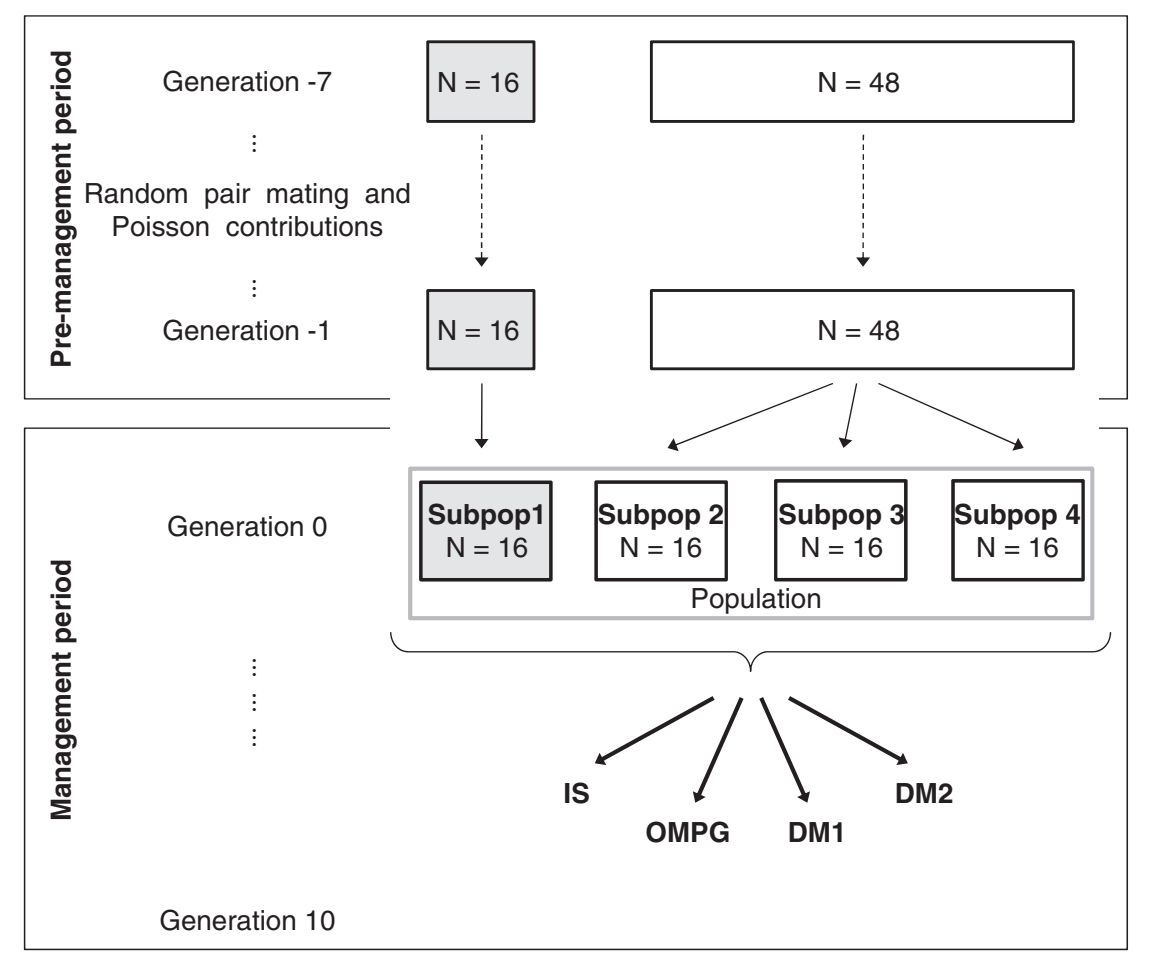

Figure 1 Experimental design during the pre-management and management periods. This full scheme was replicated three times (corresponding to the three experimental replicates). IS, isolated subpopulations; OMPG, one-migrant-per-generation method; DM1, dynamic method 1; DM2, dynamic method 2 (see text). $N$ indicates the subpopulation size. A subpopulation was intended to be more inbred and differentiated from the others, and this corresponds to the shaded square.

subpopulation was more inbred than the others and there was more differentiation between this highly inbred subpopulation and the others than between any two of the less inbred subpopulations. Four males and four virgin females were taken from each of these 32 vials to four structured populations, which would be subsequently subjected to four different treatments (see below). This guaranteed that all treatments were started from the same base and shared the same initial genealogy. All four subpopulations were maintained thereafter in eight vials (with a single couple each). Individual genealogies were recorded throughout the whole experiment.

Management experiment: From generations 0 to 10, three replicates of four structured populations (with four subpopulations each) were managed under four different strategies using the software METAPOP (PérezFigueroa et al., 2009).

Isolated subpopulations: Each subpopulation was managed separately with no exchange of individuals between them. Numbers of progeny contributed by parents and mating design within subpopulations were optimized after the minimum-coancestry criterion (see optimization protocol below). Thus, monogamous mating couples and their contributions to progeny were those that minimize the average coancestry (kinship) and actual inbreeding of the progeny.

OMPG scheme: Each population was managed as for the IS scheme above. However, each generation of every subpopulation sent a descendant and received a descendant from another randomly chosen subpopulation.

Dynamic method 1: Mating pairs and their contributions to progeny, numbers of migrants and their subpopulations of origin and destiny were optimized after the model developed by Fernández et al. (2008). The objective was to achieve the lowest average coancestry maintaining the same level of inbreeding as that with OMPG. For the sake of a fair comparison with the OMPG method, the maximum average number of migrants allowed per subpopulation and generation was one (that is the total number of exchanges in the population per generation was the same for the OMPG method and the dynamic method 1 (DM1)). In addition, the optimization criterion followed was to establish contributions that minimize the overall population coancestry, but keeping the same inbreeding level as that achieved in the corresponding generation with the OMPG strategy.

Dynamic method 2: This was identical to DM1 except that the optimization criterion was to achieve the lowest average coancestry maintaining a lower inbreeding level than that with the OMPG strategy. As theoretical results indicate that an excessive restriction in the level of inbreeding increases the average coancestry of future generations (Fernández et al., 2008), this restriction was adjusted progressively. In the first generation of management, the maximum inbreeding level allowed under the dynamic method 2 (DM2) was $98.5 \%$ of that achieved with the OMPG strategy. This percentage was 
decreased each generation by $1.5 \%$ points until the seventh generation (when it was $89.5 \%$ of that for OMPG), and kept constant $(89.5 \%)$ thereafter.

\section{Optimization protocol}

The optimization protocol was implemented by the program METAPOP (Pérez-Figueroa et al., 2009). Monogamous mating was implemented in all cases and a mate selection algorithm was applied. This optimization algorithm looks simultaneously for the optimal partner for each individual and the optimal contribution to progeny from the resulting mating pair (see Fernández et al., 2001).

For isolated subpopulations (IS) and OMPG schemes, independent optimizations were performed for each subpopulation every generation, whereas in the case of the DM1 and DM2, the optimization protocol was performed for the structured population as a whole (for details on the optimization algorithm, see Supplementary Appendix A1 in online supplementary material).

An important practical aspect to be mentioned is that, if one or more matings or expected contributions could not be fulfilled (because of mating failures or lack of the required progeny), the optimization program was re-run, as the software permits including as many restrictions as necessary in the optimization procedure. Additional details on the optimization algorithms can be found in Pérez-Figueroa et al. (2009) and the METAPOP user guide available in the web page http://webs.uvigo.es/ anpefi/metapop/.

\section{Simulations of the management schemes}

The software METAPOP was also used to perform simulations of the expected outcome from the four management strategies. The observed matrix of pedigree relationships between individuals at generation zero for each of the three replicates was introduced as input of the program. Then, the four management procedures were simulated over 10 generations. These simulations are assumed to give the expected future results in inbreeding and coancestry if all expected matings and all expected contributions and migrations were fulfilled over the whole period of management. Note that, for a given starting point (coancestry matrix), iterations of the simulations would give the same result, because the optimal combination of mating couples, contributions and migrations is unique and the solver reaches (if done correctly) the same solution (this was checked). For completeness, the IS and the OMPG scenarios were also run without minimumcoancestry optimization, that is assuming pedigrees are not available and, therefore, random mating between pairs and Poisson's contributions.

\section{Estimation of fitness}

Two estimates of fitness components were computed throughout the management experiment: couple success (cs) rate, which is a measure of mating success, and competitive fitness ( $c w)$, which is a measure of overall fitness after mating including fecundity of the females and egg-to-adult viability in competition with a marker stock.

Couple success rate: Each generation of the cs rate of each subpopulation was computed as the number of couples able to leave offspring divided by the total number of couples in the subpopulation,

cs $=$ number of successful couples/total number of couples.

Competitive fitness: In generations $0,2,4,6,8$ and 10, a measure of $c w$ was recorded for each subpopulation as the proportion of wild progeny emerged from a vial in which fertilized wild and marker stock females were allowed to lay eggs together. From each of the eight subpopulation vials, three virgin females and three males were taken and a circular mating scheme was carried out in which the females from each vial were mated to the males from the next one. Simultaneously, the same number of matings between virgin $C y L^{2}$ females and $C y L^{2}$ males were carried out. After $48 \mathrm{~h}$, a wild female and a $C y L^{2}$ female were put into the same vial and they were allowed to lay eggs for 5 days. The number of wild and $C y L^{2}$ offspring per vial was recorded after complete emergence, and $c w$ was computed from the ratio of wild to total individuals in the progeny, that is

$c w=$ number of wild offspring/total number of offspring.

The above estimate is a multitrait measure (RodríguezRamilo et al., 2007), including the possibility that the females might or might not be pregnant, the egg production of the pregnant females and the egg-to-adult viability of the progeny in competition with the marker stock. A global measure of fitness was also computed as the product of the two components, $W=c s \times c w$.

Statistical test were carried out with SPSS Statistics v. 17.

\section{Microsatellite loci analysis}

Neutral genetic diversity was evaluated at generations 0 , 5 and 10 by means of the analysis of gene and allele diversity of microsatellite loci. In order to analyze at least as many individuals as the population size, about 20 flies from each subpopulation, replicate and generation (that is a total number of 2251 individuals) were stored at $-20^{\circ} \mathrm{C}$. Genomic DNA was extracted by the Chelexbased DNA extraction protocol (Estoup et al., 1996) and nine microsatellite loci were amplified by multiplex PCR and analyzed: AC002446, AC004641 (Harr and Schlötterer, 2000), Dm1639-TC (Bachtrog et al., 2000) (located at the right arm of chromosome II), 3L9222187ca (located at the left arm of chromosome III), 3R1302339ga, 3R16177365gt, $3 R 22473342 \mathrm{gt}, 3 R 24298455 \mathrm{ca}$ and $3 R 11178343 \mathrm{ga}$ (located at the right arm of chromosome III) (Kauer and Schlötterer, 2004). These nine markers are polymorphic (with an average of seven alleles per marker; range 3-14) and are located on non-coding regions with a high recombination rate to avoid that they may be affected by linked selection at flanking coding regions. (Characteristics for each analyzed microsatellite and multiplex PCR conditions can be seen in online supporting information; Supplementary Tables A1-A3.) The PCR products were separated on an ABI PRISM 3700 automated DNA sequencer using ROX-400 as a ladder in order to determine the size of each PCR product with the Genemapper 4.0 software (Applied Biosystems, Inc., Carlsbad, CA, USA).

\section{Results}

Figure 2 shows the population average inbreeding coefficient (a) and the average global population coancestry 

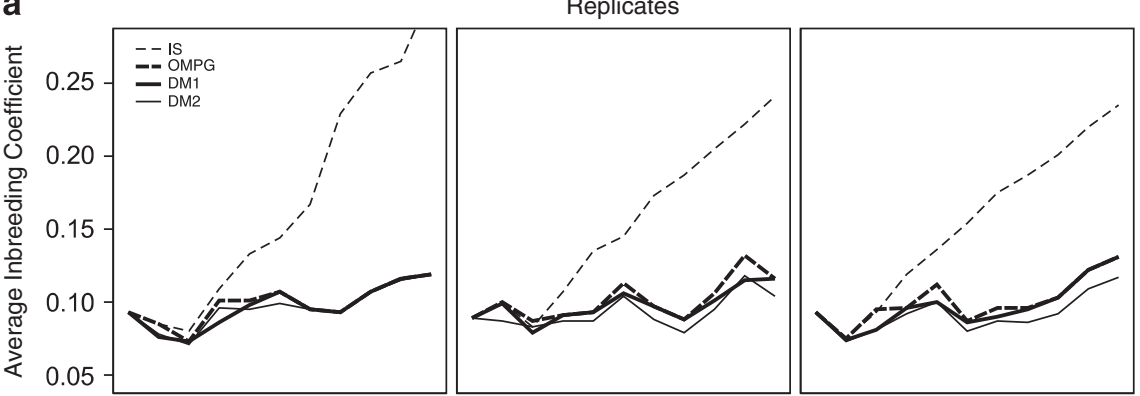

b
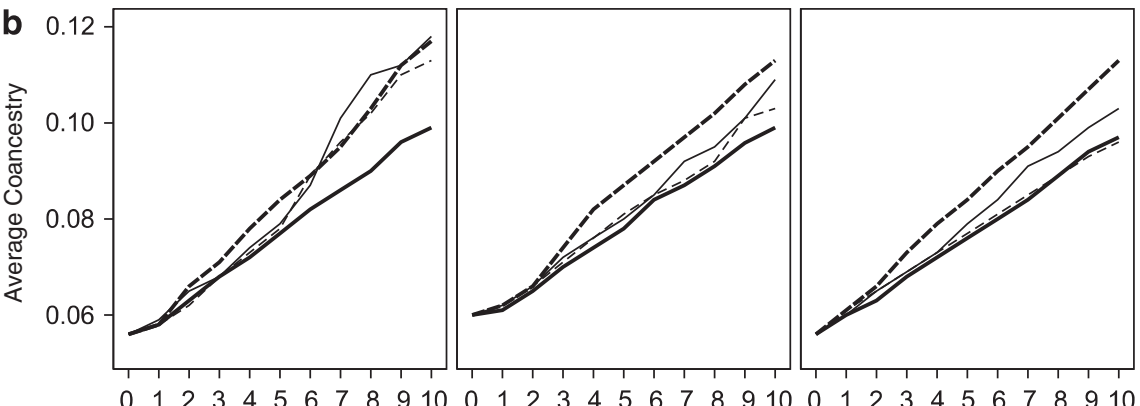

Generation

Figure 2 (a) Observed population inbreeding coefficient and (b) global coancestry for the three experimental replicates for each management strategy. IS, isolated subpopulations; OMPG, one-migrant-per-generation method; DM1, dynamic method 1; DM2, dynamic method 2 (see text).

(b) for the four management methods and the three experimental replicates. Figure 3 shows the corresponding simulation results averaged over the three simulated replicates (simulation results for each replicate separately are shown in Supplementary Appendix Figure A1). The experimental average inbreeding coefficient (Figure 2a) was significantly larger than the simulated one (Figure 3a) for all four management methods $(P=0.05$ for a non-parametric one-tailed Kolmogorov-Smirnov $\mathrm{Z}$ test at generation 10). The experimental average global coancestry at generation 10 (Figure 2b) was also higher than the simulated one (Figure $3 b$ ) for all methods, but only significant for IS and OMPG. This higher observed inbreeding and coancestry than expected is due to the unavoidable failures in the expected matings and contributions occurred in the experiment. Eventually, some couples failed in producing offspring (see cs rate in Figure $4 \mathrm{~d}$ ) or their offspring was not enough according to the minimum-coancestry rule. When this happens, it would always imply a somewhat reduced control on coancestry and inbreeding and a slight reduction in effective population size.

As expected, the IS scheme showed a substantially higher increase in inbreeding relative to the methods allowing for migration among subpopulations, for which inbreeding was controlled. Although the expectation would be that the IS method would also produce the lowest average coancestry (see simulation results in Figure $3 b$ ), the DM1 actually produced the same or a lower one in the three replicates (Figure 2b). The DM1 was aimed at producing the same inbreeding coefficient as the OMPG scheme, but a lower average coancestry. This was the result obtained by the simulations (Figure 3), and it was also achieved in the experiment (Figure 2).

The DM2 was aimed at producing both lower inbreeding and lower coancestry than the OMPG system, and this was generally the case for the whole experiment. However, the restricted inbreeding of the DM2 produced a sudden increase in coancestry at about generation 6, both in the simulations and the experiment (Figures $2 b$ and $3 b)$. For this reason, and in order to reduce the observed increase in overall coancestry, inbreeding was adjusted from generation 6 in replicate $A$ to be equal to that for OMPG (rather than smaller).

For completeness, Figure 3 also shows simulation results for the IS and OMPG methods assuming that minimum-coancestry optimization is not performed (IS$\mathrm{R}$ and OMPG-R, respectively). Clearly, the global average coancestry and inbreeding are substantially larger than when the optimization is performed. Therefore, the OMPG method applied without the knowledge of pedigree data has a limited value in restricting coancestry and inbreeding.

Figure 4a shows the observed average inbreeding for each of the subpopulations separately. One of the subpopulations (thick line) had intentionally larger inbreeding and differentiation with the others because it had been maintained separately from the rest in the pre-management period. This difference was removed with all methods allowing for migration, but the process was slightly faster with the DM1 and DM2 than with the OMPG method. A non-parametric one-tailed Kolmogorov-Smirnov $Z$ test showed that the difference in inbreeding between the highly inbred subpopulation and the rest was non-significant from generations 5 (for DM1) and 6 (for DM2), whereas it was non-significant only from generation 9 for the OMPG method (see probability values in online supporting information; Supplementary Table A4).

Note that for the OMPG method, the number of migrants coming in and out from the highly inbred subpopulation was exactly one for all generations and 


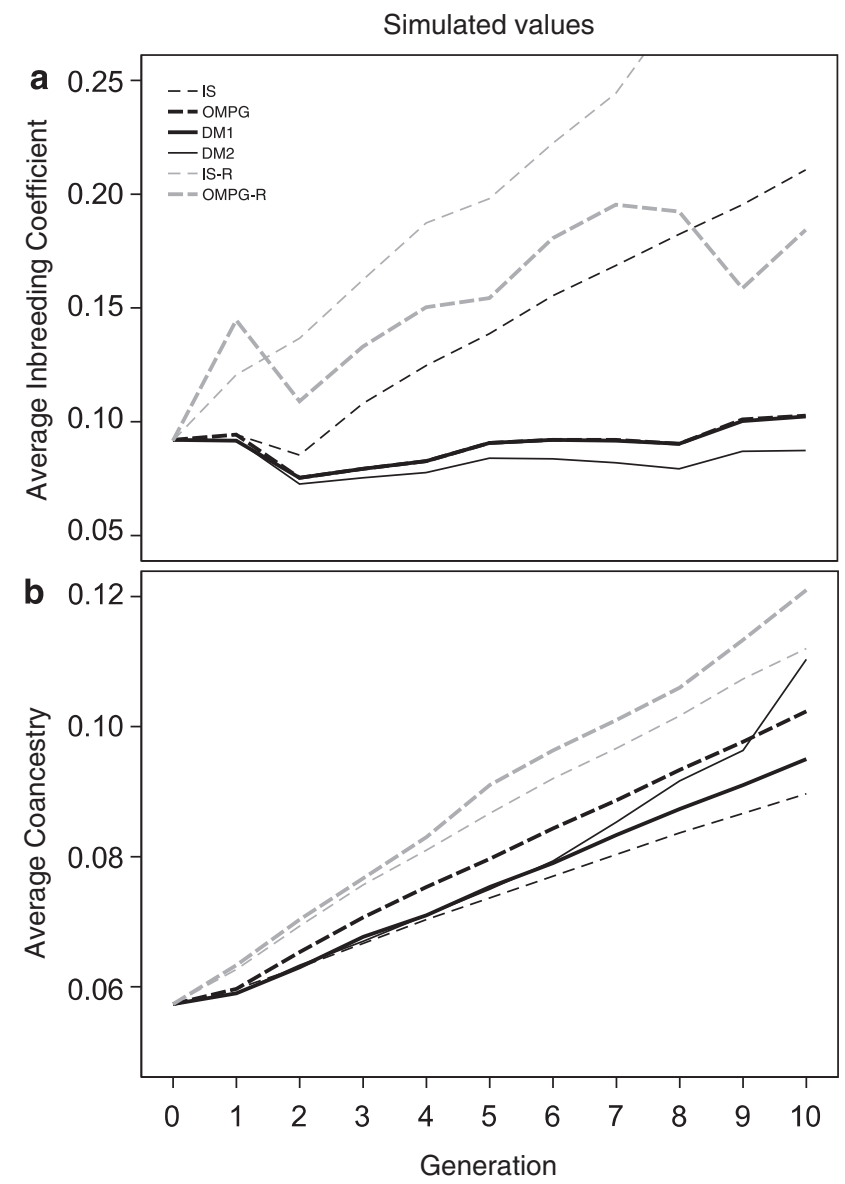

Figure 3 (a) Simulated population inbreeding coefficient and (b) global coancestry averaged over three simulation replicates for each management strategy. Black lines: IS, isolated subpopulations; OMPG, one-migrant-per-generation method; DM1, dynamic method 1; DM2, dynamic method 2. Grey lines: IS-R, isolated subpopulations without management; OMPG-R, subpopulations without management with one-migrant-per-generation migration.

subpopulations, whereas for the DM, the number was also one on average per generation for the whole set of subpopulations, but the actual number varied across subpopulations and generations (range between 0.25 and 2.25) depending on the optimization decisions.

Figures $4 \mathrm{~b}$ and c show the average allelic richness and average expected heterozygosity for the nine microsatellite loci analyzed at generations 0,5 and 10. The lower allelic richness and heterozygosity of the highly inbred subpopulation was restored by all methods allowing for migration, but again the recovery was faster for the DM than for the OMPG method. The difference in allelic richness and heterozygosity between the highly inbred subpopulation and the rest of the subpopulations was non-significant from generation 5 for DM1 and DM2, whereas it was non-significant only at generation 10 for the OMPG method (Supplementary Table A4).

Finally, the two measures of fitness, cs rate and cw, estimated for each subpopulation separately and all management methods is given in Figures $4 \mathrm{~d}$ and e. Again, it can be seen that the highly inbred subpopulation had the lowest fitness at generation 0 , and this difference was removed with all methods allowing for migration, this process being slightly faster with the
DM1 and DM2 than with the OMPG method. The difference in global fitness $(W=c s \times c w)$ between the highly inbred subpopulation and the rest of the subpopulations was non-significant from generation 2 for DM1 and DM2, whereas it was non-significant from generation 4 for the OMPG method (Supplementary Table A4). The low fitness of the highly inbred subpopulation for the IS scheme at the end of the experiment propitiated that this subpopulation in the first replicate could only be maintained with two pairs in generation 8 , and was lost in generation 9 .

\section{Discussion}

We have experimentally tested the efficiency of a DM for the management of conserved subdivided populations in comparison with schemes in which migration is excluded (IS scheme) or it is fixed as a randomly chosen individual per subpopulation and generation (OMPG method). The results showed that the DM manages inbreeding and coancestry more efficiently than the other simple alternatives.

For the sake of a fair experimental comparison, all methods included minimum-coancestry contributions and matings using pedigree information. The simulations shown in Figure 3, however, illustrate the outcome when the IS and the OMPG schemes are carried out without this optimization procedure (IS-R and OMPG-R, respectively). It is clear that the possibility of applying optimal contributions and matings has a huge impact in the control of inbreeding and overall coancestry in a conservation program. It should also be noticed that allowing for random migration without optimal contributions (OMPG-R) has a limited value in the control of inbreeding. Given this prior assumption, we focused on the following differences between methods: (1) the absence (IS) or presence (OMPG, DM) of gene flow; (2) for DM, the optimal contributions and matings were obtained by using the whole population pedigree, whereas for IS and OMPG the optimization was only achieved within subpopulations; (3) the amount of migration was the same for OMPG and DM, but the pattern of migrations was random for the former, whereas it was optimized for the latter.

From theoretical arguments and computer simulations (see Figures 2 and 3), it is expected that complete isolation (IS scheme) will lead to the highest inbreeding and the lowest coancestry among all methods contrasted. Interestingly, the DM1 produced a lower average coancestry than the IS scheme in two out of the three replicates (Figure $2 b$ ). The reason is that, in practice, death of individuals by inbreeding depression, failures of mating pairs and the consequent loss of variation will increase the theoretical minimal coancestry achieved by complete isolation. In addition, the DM1 procedure was aimed at giving a minimal coancestry controlling the inbreeding to a value as large as that for the OMPG method. This was achieved along the whole experiment (Figure 2).

The presence of gene flow between subpopulations was shown to be an efficient way for the control of inbreeding and a restoration of genetic diversity and fitness (Figure 4) in the highly inbred subpopulation. Maintenance of isolated small populations for a long time before an extensive exchange of genetic material can 
Management Strategy

IS

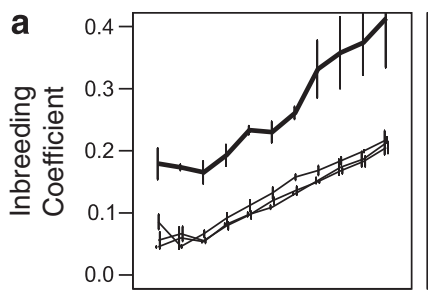

b

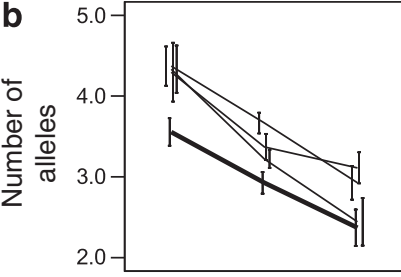

C
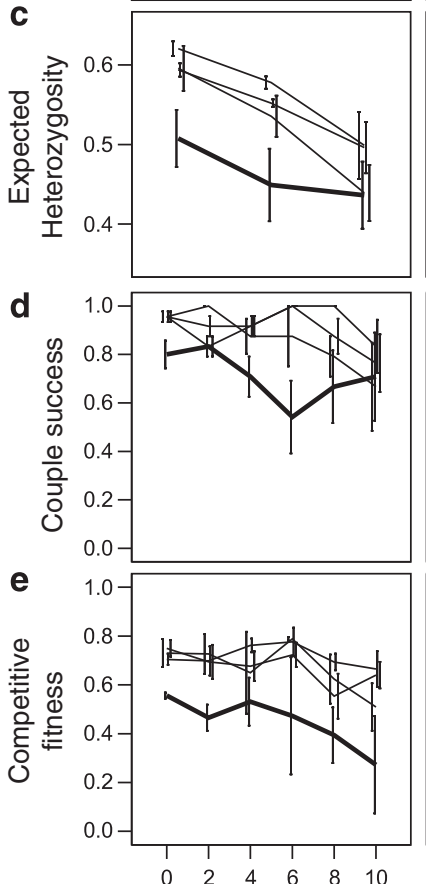

OMPG
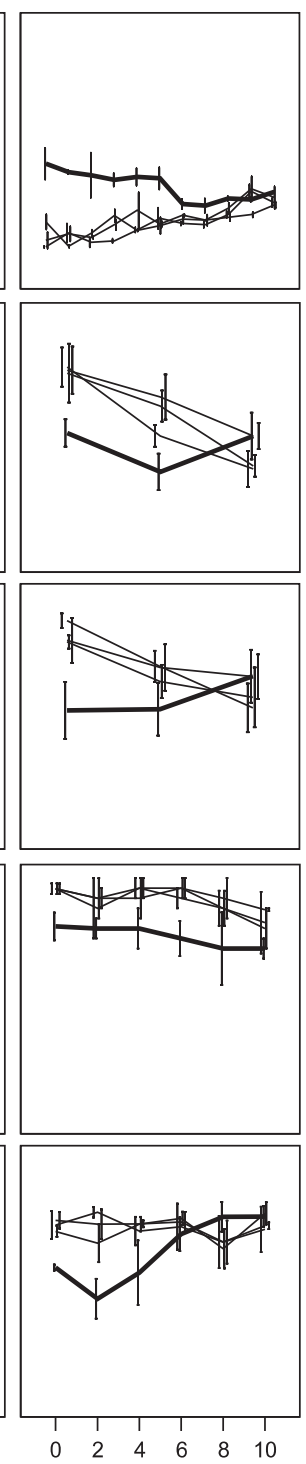

DM1
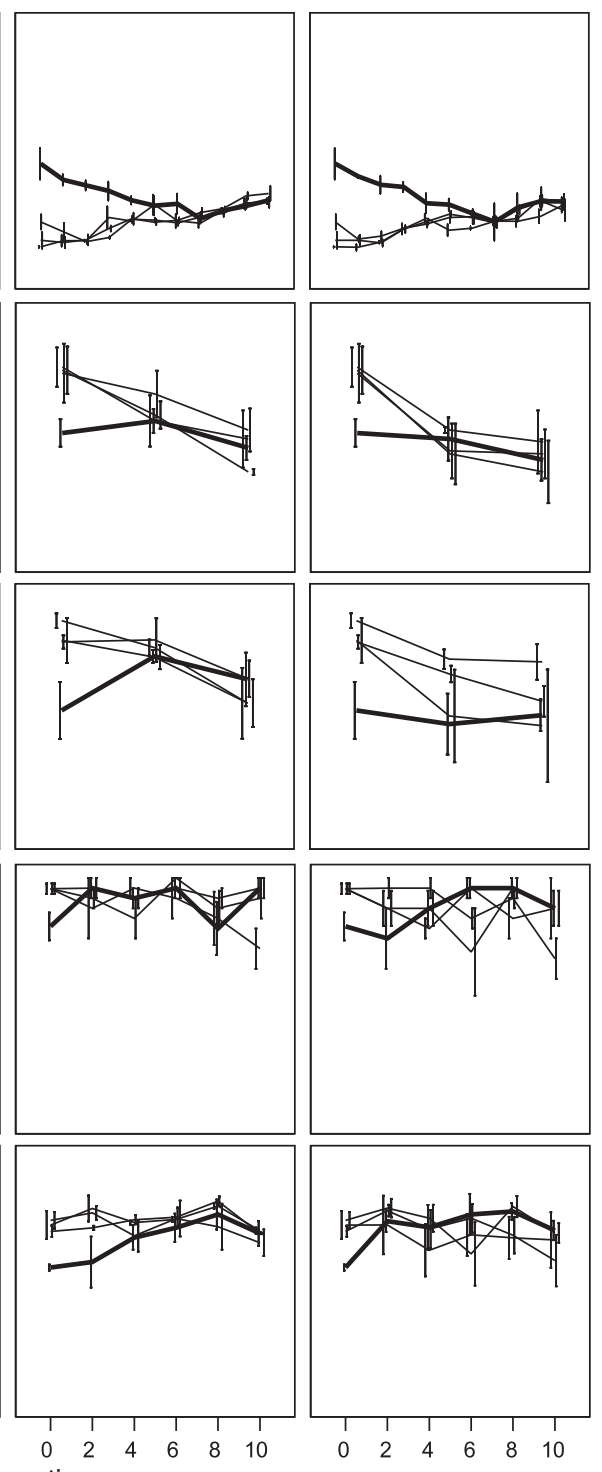

Figure 4 Subpopulation parameters averaged over replicates for each management strategy (thick line for the highly inbred subpopulation and thin lines for the other subpopulations). (a) Subpopulation inbreeding coefficient, (b) number of alleles, (c) expected heterozygosity, (d) couple success rate (cs) and (e) competitive fitness (cw). Bars indicate one s.e.

be successful in experimental species (Margan et al., 1998), but is arguably less practical in real situations, especially in animals. The decision of mixing or not subpopulations when starting or managing a captive conservation program is always under debate. Some authors propose to keep the observed structure in the nature in particular situations (Smith et al., 2009), whereas other suggests joining all populations to reduce the probability of extinction related to inbreeding depression (Miller et al., 2009). The option should be determined studying the particular characteristics of each species and population. Notwithstanding, we should recall that the DM can cope with both scenarios. If there is a particular interest in keeping groups, we can ban the migrations or impose a restriction on the level of differentiation between subpopulations $\left(F_{\mathrm{ST}}\right)$. Notice that, even when migration is not allowed, DM yields better results (higher levels of global genetic diversity) than isolated populations by accounting for between population relationships. In general, keeping ' $e x$ situ' populations subdivided could be more related to logistic/technical issues than to genetic purposes and, consequently, adequate scheme of exchanges between groups is very important.

The impact of the optimization of the migration pattern was smaller. Even so, this subtle difference made it possible that the DM1 and DM2 produced lower coancestry, lower or equal inbreeding, and a slightly faster restoration of the neutral genetic diversity and fitness of the highly inbred subpopulation relative to the OMPG scheme (Figure 4). The reasons are threefold. First, OMPG assumes constant contributions of each 
subpopulation, that is each subpopulation produces the same number of progeny to the next generation $(N)$ with the peculiarity that one offspring will be translocated to another subpopulation and another offspring will arrive from elsewhere. In contrast, the DM schemes can penalize/favor particular subpopulations because of their relationships with the others. Therefore, not necessarily each subpopulation contributes with $N$ progeny to the next generation. Second, OMPG forces the same migration rate from/to every subpopulation, whereas the DM schemes are able to detect 'peculiar' populations, redirecting the migration flow to/from them. Finally, particular individuals' contributions are decided independently within each subpopulation for the OMPG method, not accounting for the relationship between subpopulations. In contrast, under the DM schemes, contributions of individuals are influenced by their coancestry with individuals from all subpopulations, optimizing the global genetic diversity even if no migration is allowed.

The strict balance between controlling inbreeding and optimizing coancestry simultaneously is well illustrated by the results obtained with the DM2. This was aimed at giving a minimal coancestry and a level of inbreeding lower than that of OMPG. Both in the simulations and the experiment, the method worked well until around generation 6 when the low inbreeding maintained triggered a substantial increase in coancestry (Figures 2 and 3), although for the experiment, this never reached the levels of OMPG in the period considered. The ultimate reason for this sudden increase in coancestry can be illustrated as follows. If the restriction applied on inbreeding is too strong, the optimal breeding scheme deduced by the optimizing algorithm could be achieved by, say, the breeding of a single couple, in which the least-related male and female will be chosen as parents of the next generation. The progeny will show the lowest inbreeding, but the global coancestry will obviously increase, as all progeny comes from the same single couple.

From a genetic point of view, the chances of success of a conservation program depend on the census size and the original genetic diversity available. The management methods considered in this paper, particularly the DM, are just tools that take advantage of the availability of pedigree information to minimize the unavoidable loss of genetic diversity that will occur because of genetic drift in a small population. The aim of the method is to increase the actual effective population size of the population, thus minimizing the impact of genetic drift and restricting the increase in local inbreeding to any desired value by adjusting the amount and pattern of migration among subpopulations.

The balance between a desire for maintaining more global gene diversity at the expense of more local inbreeding, or vice versa, is illustrated by the different objectives and outcomes of DM1 and DM2 presented in this paper. If local inbreeding is intended to be highly restricted in order to avoid the deleterious consequences of local inbreeding depression, this will imply an overall cost in global diversity (an increase in overall coancestry) and, therefore, a reduction in the overall evolutionary potential of the system (for example a reduction in the number of allelic variants maintained in different subpopulations). The decision for setting up this balance between global coancestry and local inbreeding will depend on the particular situation.

The application of the DM (in general of the minimumcoancestry optimization) requires that matings can be manipulated and, therefore, it is restricted to captive populations of a restricted number of animal species. More possibilities of application can be envisioned, however, for plant species, regarding, for example, the conservation of germplasm collections. When mating manipulation is feasible, the DM can deal with most particular situations (overlapping generations, polygamy and so on) In our experiment, discrete generations and monogamy were restricting situations to be included in the model. Clearly, if polygamy and overlapping generations were allowed, the degrees of freedom for potential crosses and contributions would be increased and the DM would be expected to produce more efficient results.

The different management alternatives were implemented by the METAPOP software (Pérez-Figueroa et al., 2009). This software allows for carrying out simulations of the expected outcomes of a management program starting from a given pedigree structure. This is very useful from a manager's perspective, because simulations of any future number of generations can be an aid in taking decisions for the current one. Yet, the simulations assume that all matings and their contributions are fulfilled, which is often not the case in practice. When these failures occur, the optimization program has to be re-run including all restrictions incurred. This is another useful tool of the METAPOP software, which was often used in the experiment (particularly in the IS scheme) whenever the expected mates had no progeny or the required number of progeny established by the minimum-coancestry criterion was not achieved. Even so, the experimental levels of inbreeding and coancestry were generally higher than the simulated ones (cf. Figures 2 and 3).

Regarding the consequences of management methods on the fitness of populations, we should differentiate the short- and the long-term horizon. In the first generations, extinction risk for a population is more dependent on the effect of inbreeding depression. DM can control the levels of inbreeding and, thus, will help to increase the probability of survival of the population. In the long term, the fate of the program will be determined by the amount of diversity lost (related to evolutionary potential) and by the accumulation of deleterious mutations. Theoretically, the first threat can be minimized by subdividing the population (but think of the short-term problems). However, DM is able to maintain high levels of both gene and allelic diversity for quite long periods (see Fernández et al., 2008).

The importance of the accumulation of mutations will depend on the mutational model assumed. It is true that all methods, which tend to equalize the number of offspring, will accumulate more mutations than methods in which selection acts, but some simulation and experimental studies have shown that the disadvantage is not important even after a large number of generations (Rodríguez-Ramilo et al., 2007).

Adaptation to captivity is another important issue in captive programs. Methods based on the minimization of coancestry avoid adaptation to captivity, an added benefit of the DM strategy (Fernández and Caballero, 2001). 
In many conservation or breeding programs, the genealogy is unknown, especially for the generations before the start of the program. In these scenarios, the use of molecular markers may help in the management by replacing the pedigree-based coancestry matrix with a matrix calculated from the molecular information. The ability/efficiency of molecular information to complement/replace pedigree information in conservation programs was studied by Fernández et al. (2005). They used the raw molecular coancestry in a maybe unrealistic scenario in which markers have in linkage equilibrium in the base (founders) population. There are more precise coancestry estimators (for a review, see, for example, Fernández and Toro, 2006) and the number of available markers is rising quickly, so molecular tools are each day more powerful. Regardless, in most captive programs the problem is not to determine coancestries between individuals, but reduces to paternity analysis (in which all animals can be identified and there is a small group of potential parents), which is simpler and requires a smaller number of markers. Once the parentsoffspring relationships are determined, the pedigree is reconstructed and used for the management in the classical way.

\section{Conflict of interest}

The authors declare no conflict of interest.

\section{Acknowledgements}

We are indebted to Lucía Alvariño, Raquel Sampedro, Pilar Alvariño and Nieves Santamaría for technical help with the flies, and to two anonymous referees for useful comments. This work was funded by Ministerio de Ciencia e Innovación and Fondos Feder (CGL2006-13445C02/BOS and CGL2009-13278-C02). The research contract of V Ávila was financed by Spain's Ministerio de Ciencia e Innovación (Juan de la Cierva Program).

\section{References}

Allendorf FW, Luikart G (2007). Conservation and the Genetics of Populations. Blackwell Publishing: Malden, MA, USA.

Bachtrog D, Agis M, Imhof M, Schlötterer C (2000). Microsatellite variability differs between dinucleotide repeat motifs-evidence from Drosophila melanogaster. Mol Biol Evol 17: 1277-1285.

Ballou J, Lacy R (1995). Identifying genetically important individuals for management of genetic variation in pedigreed populations. In: Ballou JD, Gilpin M, Foose TJ (eds). Population Management for Survival and Recovery. Columbia University Press: New York. pp. 76-111.

Branch LC, Clark AM, Moler PE, Bowen BW (2003). Fragmented landscapes, habitat specificity, and conservation genetics of three lizards in Florida scrub. Conserv Genet 4: 199-212.

Caballero A, Santiago E, Toro MA (1996). Systems of mating to reduce inbreeding in selected populations. Anim Sci 62: 431-442.

Caballero A, Toro MA (2000). Interrelations between effective population size and other pedigree tools for the management of conserved populations. Genet Res 75: 331-343.

Caballero A, Toro MA (2002). Analysis of genetic diversity for the management of conserved subdivided populations. Conserv Genet 3: 289-299.
Estoup A, Largiader CR, Perrot E, Chourrout D (1996). Rapid one-tube DNA extraction for reliable PCR detection of fish polymorphic markers and transgenes. Mol Mar Biol Biotech 5: 295-298.

Fernández J, Caballero A (2001). Accumulation of deleterious mutations and equalization of parental contributions in the conservation of genetic resources. Heredity 86: 480-488.

Fernández J, Toro MA (2006). A new method to estimate relatedness from molecular markers. Mol Ecol 15: 1657-1667.

Fernández J, Toro MA, Caballero A (2001). Practical implementations of optimal management strategies in conservation programmes: a male selection method. Anim Biodiv Conserv 24: $17-24$

Fernández J, Toro MA, Caballero A (2003). Fixed contributions designs versus minimization of global coancestry to control inbreeding in small populations. Genetics 165: 885-894.

Fernández J, Toro MA, Caballero A (2004). Managing individuals' contributions to maximize the allelic diversity maintained in small, conserved populations. Conserv Biol 18: 1358-1367.

Fernández J, Toro MA, Caballero A (2008). Management of subdivided populations in conservation programs: development of a novel dynamic system. Genetics 179: 683-692.

Fernández J, Villanueva B, Pong-Wong R, Toro MA (2005). Efficiency of the use of molecular markers in conservation programmes. Genetics 170: 1313-1321.

Frankham R, Ballou JD, Briscoe DA (2002). Introduction to Conservation Genetics. Cambridge University Press: Cambridge.

Haig SM, Ballou JD, Derrickson SR (1990). Management options for preserving genetic diversity: reintroduction of Guam Rails to the wild. Conserv Biol 4: 290-300.

Harr B, Schlötterer C (2000). Long microsatellite alleles in Drosophila melanogaster have a downward mutation bias and short persistence times, which cause their genome-wide underrepresentation. Genetics 155: 1213-1220.

Kauer MO, Schlötterer C (2004). An analysis of genetic differentiation among assortatively mating Drosophila melanogaster in Zimbabwe. J Evol Biol 17: 493-500.

Kimura M, Crow JF (1963). On the maximum avoidance of inbreeding. Genet Res 4: 399-415.

Margan SH, Nurthen RK, Montgomery ME, Woodworth LM, Lowe LM, Briscoe DA et al. (1998). Single large or several small? Population fragmentation in the captive management of endangered species. Zoo Biol 17: 467-480.

Meuwissen THE (2007). Operation of conservation schemes. In: Oldenbroek K (ed). Utilisation and Conservation of Farm Animal Genetic Resources. Wageningen Academic Publishers: Wageningen, The Netherlands. pp. 167-193.

Miller KA, Chapple DG, Towns DR, Ritchie PA, Nelson NJ (2009). Assessing genetic diversity for conservation management: a case study of a threatened reptile. Anim Conserv 12: 163-171.

Mills LS, Allendorf FW (1996). The one-migrant-per-generation rule in conservation and management. Conserv Biol 10: 1509-1518.

Nei M (1973). Analysis of gene diversity in subdivided populations. Proc Natl Acad Sci USA 70: 3321-3323.

Pérez-Figueroa A, Saura M, Fernández J, Toro MA, Caballero A (2009). METAPOP - a software for the management and analysis of subdivided populations in conservation programs. Conserv Genet 10: 1097-1099.

Robertson A (1964). The effect of nonrandom mating within inbred lines on the rate of inbreeding. Genet Res 5: 164-167.

Rodríguez-Ramilo ST, Toro MA, Martínez P, Castro J, Bouza C, Fernández J (2007). Accuracy of pairwise methods in the reconstruction of family relationships, using molecular information from turbot (Scophthalmus maximus). Aquaculture 273: $434-442$. 
Saura M, Pérez-Figueroa A, Fernández J, Toro MA, Caballero A (2008). Preserving population allele frequencies in ex situ conservation programs. Conserv Biol 22: 1277-1287.

Smith AL, Gardner MG, Fenner AL, Bull CM (2009). Restricted gene flow in the endangered pygmy bluetongue lizard (Tiliqua adelaidensis) in a fragmented agricultural landscape. Wildl Res 36: 466-478.

Sonesson AK, Meuwissen THE (2000). Mating schemes for optimum contribution selection with constrained rates of inbreeding. Genet Sel Evol 32: 231-248.

Storfer A (1999). Gene flow and endangered species translocations: a topic revisited. Biol Conserv 87: 173-180.

Toro MA, Fernández J, Caballero A (2009). Molecular characterization of breeds and its use in conservation. Livest $\mathrm{Sci}$ 120: 174-195.

Toro MA, Pérez-Enciso M (1990). Optimization of selection response under restricted inbreeding. Genet Sel Evol 22: 93-107.

Valladares-Padua C, Ballou JD, Martins CS, Cullen Jr L (2002). Metapopulation management for the conservation of Black
Lion Tamarins. In: Kleiman G, Rylands A (eds). Lion Tamarins: Biology and Conservation. Smithsonian Institution Press: Washington, D.C. pp 301-314.

Wang J (2004). Application of the one-migrant-per-generation rule in conservation and management. Conserv Biol 18: 332-343.

Wang J (2005). Monitoring and managing genetic variation in group breeding populations without individual pedigrees. Conserv Genet 5: 813-825.

Wang J, Caballero A (1999). Developments in predicting the effective size of subdivided populations. Heredity 82: 212-226.

Watanabe K, Kanagawa N, Kakioka R, Itai T, Mori S (2009). Genetic diversity and conservation units in wild and captive populations of endangered freshwater fishes: a case of Hemigrammocypris rasborella in Shizuoka, Japan. Ichthyol Res 56: 411-416.

Wright S (1931). Evolution in Mendelian populations. Genetics 16: 97-259.

Supplementary Information accompanies the paper on Heredity website (http://www.nature.com/hdy) 